\title{
Could the coronavirus be gone by the end of 2021?
}

\author{
Chang-Ju Kim (Did https://orcid.org/0000-0003-4749-5795
}

The period from 2020 to 2021 was a year dominated by the coronavirus worldwide. Since the end of 2019, the coronavirus, which originated in Wuhan, China, has taken over the world and is still ongoing. Entering the second half of 2021, human efforts to defeat the coronavirus seem to bear fruit.

Vaccinations are accelerating, and the development of a coronavirus treatment is imminent, so it seems that there is not much time left before we will be freed from the fear of the coronavirus. Each country is promoting "with Corona" as a strategic response method. In other words, it is a strategy to treat coronavirus infection like a cold. Human wisdom about the invasion of the coronavirus, such as the development of various types of vaccines and the development of treatments for coronavirus, is counterattacking. In a way, this war looks like humans will win, but the coronavirus is also resisting by creating variants. It is not yet possible to determine who will win this tough battle.

Will humanity's efforts to beat coronavirus free humans from coronavirus? The decisive situation for that judgment is gradually approaching. We have been watching the results of the clash of human wisdom and virus mutations. This suffering caused by one kind of virus makes us humble for nature rather than pride in science and medicine developed by humans. As human history proves, we hope that this fight against the coronavirus will also end in human victory. The last day of 2021 has arrived. The year we spent together with coronavirus is finally coming to an end. In the new year of 2022, I hope we can find our normal daily life.

The publication of the last papers for 2021 is now over. I would also like to thank the authors who submitted their papers, and I promise a faster and more constructive review in 2022.

Merry Christmas and a Happy New Year!

\section{CONFLICT OF INTEREST}

No potential conflict of interest relevant to this article was reported.

President of the Korean Society of Exercise Rehabilitation Department of Physiology, College of Medicine, Kyung Hee University, 26 Kyungheedae-ro, Dongdaemun-gu, Seoul 02447, Korea Email: changju@khu.ac.kr 